\title{
Endoplasmic reticulum stress activation in adipose tissue induces metabolic syndrome in individuals with familial partial lipodystrophy of the Dunnigan type
}

\author{
Maria C. Foss-Freitas ${ }^{1 * \dagger}$, Rafael C. Ferraz ${ }^{1 \dagger}$, Luciana Z. Monteiro ${ }^{2}$, Patricia M. Gomes ${ }^{1}$, Ricardo Iwakura ${ }^{3}$,
} Luiz Carlos C. de Freitas ${ }^{3}$ and Milton C. Foss ${ }^{1}$

\begin{abstract}
Background: Familial partial lipodystrophy of the Dunnigan type is one of the most common inherited lipodystrophies variables. These individuals have important metabolic disorders that cause predisposition to various diseases. In this study we aimed to demonstrate the relation between the metabolic abnormalities, inflammatory profile and the expression of genes involved in the activation of the endoplasmic reticulum stress (ERS) in subjects with FPLD.

Methods: We evaluated 14 female FPLD patients and compared with 13 female healthy individuals. The subjects were paired with their respective BMI and age and categorized into two groups: Familial partial lipodystrophy of the Dunnigan type (FPLD) and control. Patients were fasted for $12 \mathrm{~h}$ before blood collection for measurement of HbA1c, glucose, insulin, lipids and inflammatory markers. Subcutâneous adipose tissue was collected by puncture aspiration of submental region during ambulatorial surgical aesthetic procedure.

Results: We demonstrate that patients with FPLD show increased HbA1c $(p<0.01)$, fasting glucose $(p<0.002)$ and triglycerides $(p<0.005)$ while HDL/cholesterol $(p<0.001)$ was lower when compared to healthy individuals. We found that $64.2 \%$ FPLD patients had metabolic syndrome according to International Diabetes Federation definition. We also observe increased AUC of glucose $(p<0.001)$ and insulin during oGTT, featuring a frame of hyperglycemia and hyperinsulinemia, suggesting insulin resistance. Also we found hyperactivation of several genes responsible for ERS such as ATF-4 $(p<0.01)$, ATF-6 ( $p<0.01)$, EIF2a3K ( $p<0.005)$, CCT4 $(p<0.001)$, CHOP $(p<0.01)$, CALR $(p<0.001)$ and CANX ( $p<0.005)$, that corroborate the idea that diabetes mellitus and metabolic syndrome are associated with direct damage to the endoplasmic reticulum homeostasis. Ultimately, we note that individuals with lipodystrophy have an increase in serum interleukins, keys of the inflammatory process, as IL-1 $\beta$, TNF-a and IL-6 ( $p<0.05$ all), compared with healthy individuals, which can be the trigger to insulin resistance in this population.
\end{abstract}

Conclusion: Individuals with FPLD besides having typical dysfunctions of metabolic syndrome, show a hyperactivation of ERS associated with increased systemic inflammatory profile, which together may explain the complex clinical aspect of this diseases.

Trial registration HCRP no 6711/2012

Keywords: Familial partial lipodystrophy, Dunnigan type, Endoplasmic reticulum stress, Insulin resistance

\footnotetext{
*Correspondence: crisfoss@fmrp.usp.br

${ }^{\dagger}$ Maria C. Foss-Freitas and Rafael C. Ferraz contributed equally to this work

${ }^{1}$ Department of Medicine, Division of Endocrinology and Metabolism,

School of Medicine of Ribeirao Preto, University of Sao Paulo, Ribeirao

Preto, São Paulo, Brazil

Full list of author information is available at the end of the article
} 


\section{Background}

Lipodystrophy are part of a clinically heterogeneous group of inherited or acquired diseases characterized by selective or total loss of adipose tissue. Affected patients are predisposed to insulin resistance and the development of related diseases such as visceral obesity, hypertension, type 2 diabetes mellitus, metabolic syndrome, dyslipidemia, coronary artery disease and hepatic steatosis $[1,2]$. Recently, the diagnosis of lipodystrophy has been associated with increased morbidity and mortality in patients seropositive for human immunodeficiency virus (HIV) on antiretroviral treatment [3, 4]. The molecular mechanisms involved in insulin resistance and the metabolic complications presented by patients with lipodystrophy, especially in hereditary forms are not clearly understood [5]. Being essentially clinically diagnosed, these diseases are considered rare and little known, and may go unnoticed in daily clinical practice.

The Familial partial lipodystrophy of the Dunnigan type (FPLD) is the most common and well characterized genotype-phenotype between hereditary forms of lipodystrophy, it is associated with insulin resistence and can evolve with decreased glucose tolerance and development of diabetes mellitus and dyslipidemia [6]. It is an autosomal dominant disorder, resultant of mutations in the LMNA gene, in which the loss of subcutaneous fat affects the extremities, buttocks area, abdomen and torso. Fat deposits tend to accumulate on the face, neck and submental regions, supraclavicular and intra-abdominal. In women, the frequent presence of hirsutism, menstrual abnormalities, absence of obesity of the trunk and the accumulation of fat in the genital area facilitates the diagnosis $[6,7]$. Recent studies have shown that the expression of the phenotype varies according to gender, with men presenting less aggressive metabolic profile than female patients [6].

There is growing evidence that endoplasmic reticulum stress (ERS) activation is a central feature involved in the pathogenesis of a variety of diseases, including diabetes mellitus [8]. The activated ERS is responsible for inducing apoptosis to be able to contribute to insulin resistance [9]. In addition, ERS is the consequence of a lack of control in the amount of poorly folded proteins inside the ER, which, in turn, to restore homeostasis, the cell develops a response system known as depleted protein response (UPR) [10]. The cellular imbalance induced by UPR activation interferes with the calcium balance in ER affecting genes such as calreticulin (CARL) and calnexin (CANX) [11, 12]. Several mechanisms are the mechanisms by which UPR reverses ERS, including the transmembrane sensor, such as the eukaryotic 2-alpha kinase 3 translation initiation factor (EIF2AK3 or PERK), when activated by UPR, are sufficient to activate the factor of transcription activator 6 (ATF-6), resulting in a reduction of protein synthesis avoiding the accumulation of new unfolded proteins [13]. ERS plays an important role in cellular functioning, however, some studies have shown that ERS is involved in beta cell dysfunction of the pancreas, which are responsible for insulin production [14]. There are few studies evaluating ERS in patients with lipodystrophy, but recently it has been reported that accumulation of prelamin A causes mitochondrial dysfunction, endoplasmic reticulum stress and altered lipid metabolism, similar to a phenotype of premature aging [15]. On the other hand, once the involvement of ERS in lipodystrophy has been established, new drugs and interventions that are capable of reversing the insulin resistance or dyslipidemia, frequently observed in these diseases, can be investigated [16].

Despite the reduction in the amount of adipose tissue disposed through the body of these individuals, the increased prevalence of the metabolic syndrome is evident. Many studies have shown that pro-inflammatory cytokines, produced by adipocytes, reduce insulin sensitivity induced by interference in the insulin signaling pathway [17]. In addition to the inflammatory process, the activation of endoplasmic reticulum stress, particularly in adipose tissue, plays an important role in the development of insulin resistance and hyperglycemia [8].

In this study, we analyzed metabolic and inflammatory profile and also the expression of some important genes involved in the activation of ERS, which plays an important role in insulin resistance and dislipidemia, in subjects with FPLD.

\section{Methods}

\section{Individuals}

After obtaining the consent, 14 female patients with FPLD, followed at the Diabetes Outpatient Clinic, and 13 female healthy subjects paired for age and BMI, constituting the control group, were studied. Subjects with FPLD had clinical and molecular testing diagnosis as described previously [18]. The study was approved by the Research Ethics Committee (HC-FMRP-USP-Process HCRP no 6711/2012) of the Institution and all volunteer subjects gave written informed consent to participate.

\section{Inclusion criteria}

All patients in the FPLD group had androgenic phenotype, muscular hypertrophy, increased waist/hip ratio (> 0.85), breast hypotrophy, hyperinsulinemia, rounded and wide facies, fat deposition in the cervical and mandibular regions, filled supraclavicular fossa, acanthosis nigricans, menstrual irregularity, DM and hyperlipidemia. The FPLD diagnosis was confirmed by molecular testing that was previously described [18]. 
Control group volunteers were selected aleatory in the outpatient clinic, they should not present the above characteristics but should be matched for age and BMI with FPLD subjects.

\section{Exclusion criteria}

Patients who had chronic DM complications in advanced stages (proliferative retinopathy, chronic renal failure, autonomic neuropathy, and acute myocardial infarction or history of peripheral vascular accident less than 6 months), gestation, chronic or acute liver diseases, cancer and high levels of plasma LDL cholesterol (above $150 \mathrm{mg} / \mathrm{dL}$ ) were not enrolled in the study.

\section{Use of medications}

All subjects in the FPLD group were using metformin and statins to control glycemic and cholesterol levels. Healthy subjects in the control group were not using any medication.

\section{Data collect}

Patients and healthy volunteers were submitted initially to clinical evaluation and body composition, weight, height and waist circumference, as well as oGTT. There were also collected blood samples for glucose, insulin and lipid profile measurements, for this procedure patients and volunteers were fasted for $8 \mathrm{~h}$.

\section{Specific laboratory determinations}

A blood sample was collected after an overnight fast of $8 \mathrm{~h}$. Plasma glucose levels were measured using COBAS INTEGRA 400 plus (Roche, USA). Fasting insulin levels were measured using a radioimmunoassay by Immulite I (Siemens, USA). Fasting lipids were analyzed, and for the present study serum levels of total cholesterol, LDL, HDL and triglyceride using COBAS INTEGRA 400 plus (Roche, USA).

\section{Insulin resistance evaluation and systemic inflammatory state}

Serum levels of CRP were evaluated for systemic inflammatory evaluation, as well as HbA1c and lipids and total fractions. At the same time, serum insulin and fasting blood glucose levels were measured to calculate the HOMA index. The method is based on basal glycemia and fasting insulin to assess insulin resistance (HOMAIR) and $\beta$-cell function $(\% \beta)$. The following equations were used for this calculation:

$$
\begin{aligned}
& \text { HOMA- } \beta=\frac{20 \times \text { Basal insulin }(\mu \mathrm{U} / \mathrm{mL})}{\text { Fasting glycemia }(\mathrm{mmol} / \mathrm{L})-3.5} \\
& \text { HOMA-IR }=\frac{\text { Fasting glycemia }(\mathrm{mmol} / \mathrm{l}) \times \text { Basal insulin }(\mu \mathrm{U} / \mathrm{mL})}{22.5}
\end{aligned}
$$

\section{Oral glucose tolerance test (oGTT)}

For oGTT, the individuals were seen in the hospital in the morning after $10 \mathrm{~h}$ of fasting. Blood samples were collected prior to the administration of $75 \mathrm{~g}$ of anhydrous glucose dissolved in $300 \mathrm{~mL}$ of water orally (time $0^{\prime}$ ) and then every $30 \mathrm{~min}$ (times $30^{\prime}, 60^{\prime}, 90^{\prime}$ and $120^{\prime}$ ) for dosing of serum glucose and insulin.

\section{Quantification of serum cytokines}

Blood samples were collected in BD Vacutainer ${ }^{\circledR} \mathrm{SST}^{\mathrm{TM}}$ II Advance tubes, centrifuged, the serum was separated and frozen in aliquots at $-70{ }^{\circ} \mathrm{C}$ for subsequent dosing. Serum Cytokines TNF- $\alpha$, IL- 6 e IL- $1 \beta$ they were quantified using enzyme immunoassay (ELISA) (BD Biosciences, New Jersey, EUA). Individuals in the FPLD and Control groups were fasted for $8 \mathrm{~h}$ before blood was withdrawn.

\section{Puncture of the subcutaneous fat}

The subcutaneous adipose tissue of the neck was collected by puncture during aesthetic procedure. The procedure was performed by personnel trained under local anesthesia (2\% lidocaine with epinephrine). After antisepsis with $2 \%$ chlorhexidine, puncture was performed with a $16 \mathrm{G}$ hypodermic needle $(1.6 \times 40 \mathrm{~mm})$ attached to a $10 \mathrm{~mL}$ syringe. After the needle was introduced, repeated movements were made in tangential directions with the piston of the syringe drawn to cause vacuum. Approximately $1.0 \mathrm{~mL}$ of tissue (approximately $1 \mathrm{~g}$ ) was collected which was frozen in liquid nitrogen and stored in a freezer at $-70^{\circ} \mathrm{C}$ until processing. Five patients with FPLD and four healthy individuals matched for age and BMI were submitted to the procedure. In this study we had only 5 samples of adipose tissue from FPLD patients due to the non acceptance of 10 patients to perform the biopsy to collect the sample. Prior to the procedure, all subjects were asked to fast for $8 \mathrm{~h}$.

\section{Human unfolded protein response PCR-array}

RNA extraction was performed using the aspirated material from the cervical region. Briefly, the total adipose tissue that was collected was ground and homogenized following the extraction protocol of the RNeasy Lipid Tissue Mini Kit-QIAGEN. After quantification and evaluation of RNA integrity, a pool of samples from the CTRL group $(\mathrm{n}=4)$ and the FPLD group $(\mathrm{n}=5)$ were created. To do this, he added $100 \mathrm{ng}$ of RNA from the CTRL and FPLD groups of each individual to make two sets of samples. We quantified the RNA concentration again and the following concentrations were observed: CTRL Pool $105.1 \mathrm{ng} / \mu \mathrm{L}$ and FPLD Pool $110.7 \mathrm{ng} / \mu \mathrm{l}$. Next we produced cDNA using the iScript cDNA Synthesis Kit-Bio-Rad. The cDNA was then used in the Human 
Unfolded Protein Response PCR Array-Qiagen (PAHS089Y). This first evaluation was performed as a screening, because it is a panel with 84 genes involved in unfolded protein response, which would later be validated in realtime PCR. The temperatures and times used to program the Bio-Rad unit (CFX96) were: 1 cycle -10 min $-95^{\circ} \mathrm{C}$; 40 cycles $-15 \mathrm{~s}-95^{\circ} \mathrm{C}$; Cycle $40-1 \mathrm{~min}-60^{\circ} \mathrm{C}$.

\section{Gene expression analysis}

Briefly, 5 samples of adipose tissue from the FPLD group and 4 samples from the CTRL group were collected, and total RNA was extracted as described above. We used $1 \mu \mathrm{g}$ of total RNA from each sample to produce cDNA using iScript cDNA Synthesis Kit (Bio-Rad). Quantitative PCR was performed using the SsoFastTM EvaGreen Supermix (Bio-Rad) and gene expression was quantified by real-time PCR in an CFX96 (Bio-Rad) (FPLD $\mathrm{n}=5$ and CTRL $\mathrm{n}=4$ ). Relative gene expression was normalized to GAPDH mRNA levels. The primers used in the qPCR analyzes are presented in Additional file 1: Table S1 (Primers Sequence). Specificity of amplification was tested by melting analysis. All samples were analyzed in duplicate.

\section{Statistical analysis}

All results are expressed as mean \pm SEM for metabolic experiments, gene expression and interleukin quantification experiments. $\mathrm{t}$ Student test was used to determine $\mathrm{p}$ values. Statistical significance was defined as $\mathrm{p}<0.05$.

\section{Results}

\section{Familial partial lipodystrophy of the Dunnigan type} induces metabolic changes

In our study, we observed that patients with FPLD had elevated glycosylated hemoglobin levels compared to normal subjects $(8.1 \pm 2.7 \%)$ (Fig. 1a), corroborating the results of plasma glucose levels, which also presented higher serum levels $(130.9 \pm 51.9 \mathrm{mg} / \mathrm{dL})$ compared to control subjects $(80.3 \pm 7.3 \mathrm{mg} / \mathrm{dL})$ (Table 1$)$. We also observed higher levels of triglycerides $(224.8 \pm 85.9 \mathrm{mg} /$ dL) (Fig. 1b), but no changes in LDL cholesterol levels $(131 \pm 51.6 \mathrm{mg} / \mathrm{dL})$ (Fig. 1d). We observed a slight
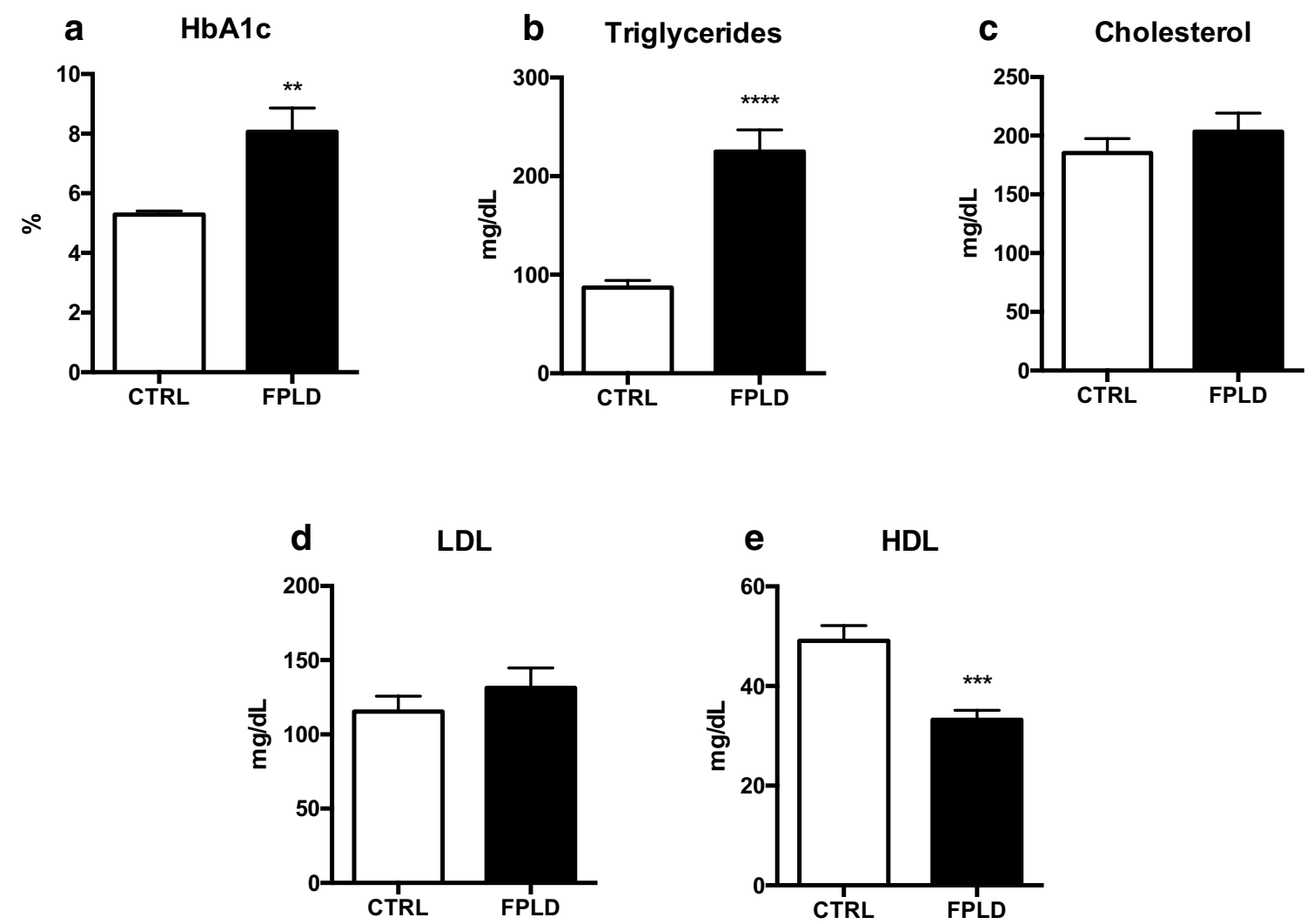

Fig. 1 Lipodystrophy induces changes in serum levels of various blood metabolites. Individuals with FPLD have high blood metabolic parameters in comparison with control subjects. a Quantification of glycated hemoglobin levels. Student's t test ${ }^{* *} p<0.01$. b Quantification of serum triglyceride levels. Student's t test ${ }^{* *} p<0.005$. c Evaluation of the amount of blood cholesterol. Student's t test (ns). d Quantification of serum LDL levels. Student's t test (ns). e Quantification of HDL levels. Student's t test ${ }^{* * *} p<0.001$. Control group $(n=13)$, FPLD group $(n=14)$ 
increase in total cholesterol $(203.4 \pm 61.0 \mathrm{mg} / \mathrm{dL})$ (Fig. 1c), followed by reduction of HDL cholesterol $(33.2 \pm 7.3 \mathrm{mg} / \mathrm{dL})$ (Fig. 1e). These data show metabolic alterations in people with FPLD, confirmed with a $64.2 \%$ prevalence of metabolic syndrome in this group. The comparison between the FPLD and the control groups showed that there were no significant differences in BMI, waist circumference and age among the members of the group, however, fasting serum insulin levels were higher in the FPLD group $(42.4 \pm 21.5 \mathrm{pmol} / \mathrm{L})$ compared to the control group $(26.3 \pm 10.3 \mathrm{pmol} / \mathrm{L})$ (Table 1$)$.

\section{Individuals with FPLD have a higher prevalence of IR}

When assessing the values found in the HOMA-IR calculation, we observed an increase in the FPLD group compared to the control group, whereas HOMA beta values were significantly lower in the FPLD group compared to the normal group (Table 1). To confirm HOMA-IR results, we evaluated the blood glucose curves of oGTT from both groups. As a result, we obtained higher levels of glucose at all times in individuals with FPLD compared to the control group (Fig. 2a), better visualized in the quantification of the area under the glucose curve (AUCg) (Fig. 2c). We also measured the insulin levels of the different groups and found that individuals with lipodystrophy had higher insulin values at 30 and $120 \mathrm{~min}$, but lower levels in the first period of oGTT (Fig. 2b), characterizing a state of hyperinsulinemia associated with loss of rapid and initial secretion of insulin. This pattern of insulin secretion resulted in an area under the insulin curve slightly lower than the control group. In addition, the FPLD group had low systemic subclinical inflammation, characterized by levels within the normal range of C-reactive protein (CRP) $(0.62 \pm 0.64 \mathrm{mg} /$ $\mathrm{dL})$, but higher than the control group $(0,20 \pm 0.20 \mathrm{mg} /$ dL) (Fig. 2d).

\section{Inflammatory profile of individuals with FPLD is altered compared to healthy subjects}

Faced with the evidence that individuals with FPLD had a subclinical inflammation, we evaluated the main interleukins responsible for systemic inflammation. The quantification of these interleukins in the serum of these individuals showed an increase in the proinflammatory cytokines, such as IL-1beta and TNF-alpha, which had high serum concentrations of FPLD individuals compared to control individuals (Fig. 3a, b). We also observed that IL-6 levels were elevated in the serum of FPLD subjects compared to healthy subjects (Fig. 3c).

\section{Subjects with FPLD present activation of ERS in adipose tissue}

To evaluate genes involved in the ERS activation machinery in the adipose tissue of patients with FPLD and normal individuals (CTRL), initially, we used the array technique in which we evaluated the expression of 84 different genes involved in Unfolded Protein Response and ERS. This experiment was performed as a screening to guide the next steps, and showed that gene expression of CCT4, CALR and CANX chaperones were increased in patients withlipodystrophy compared to the CTRL group (Additional file 2: Fig. S1). These data were confirmed by real-time PCR and other genes involved in the activation of ERS were also evaluated in order to expand the evaluation. We observed that the expression of ATF-4, ATF-6, PERK and C/EBP Homologous Protein (CHOP), were also increased in patients with lipodystrophy compared to control subjects (Fig. 4).

\section{Discussion}

In this study, we observed that patients with FPLD present disturbances in glycidic and lipid metabolism compared to healthy individuals, matched for age and BMI. We also observed insulin resistance in these individuals by both oGTT and HOMA-IR/ $\beta$, and our data indicate an increase in plasma proinflammatory interleukins in patients with FPLD. In addition, we demonstrated the presence of ERS activation in the adipose tissue of individuals with FPLD compared to controls. Thus, we sought to demonstrate the relationship between metabolic abnormalities and the inflammatory profile with the expression of ERS-related genes.

Table 1 Clinical phenotype of FPLD subjects

\begin{tabular}{lcccc}
\hline & $\begin{array}{l}\text { FPLD group } \\
\text { (mean/SD) }\end{array}$ & $\begin{array}{l}\text { Control group } \\
\text { (mean/SD) }\end{array}$ & $\begin{array}{l}\text { Normal reference } \\
\text { (mean) }\end{array}$ & $\begin{array}{l}\text { p value } \\
\text { FPLD vs. control }\end{array}$ \\
\hline Age (years) & $39.2 \pm 14.29$ & $31.3 \pm 6.9$ & - & $\mathrm{ns}$ \\
BMI (kg/m ${ }^{2}$ ) & $23.9 \pm 2$ & $23.5 \pm 1.7$ & $18.5-24.9$ & $\mathrm{~ns}$ \\
Abdominal circumference (cm) & $77.6 \pm 5.2$ & $75.3 \pm 5.5$ & 88 & $\mathrm{~ns}$ \\
Glycemia (mg/dL) & $130.9 \pm 51.9$ & $80.3 \pm 7.3$ & 100 & 0.002 \\
Insulin & $42.4 \pm 21.5$ & $26.3 \pm 10.3$ & 25 & 0.02 \\
HOMA-IR & $1.1 \pm 1.0$ & $0.4 \pm 0.2$ & $1.7-2.0$ & 0.03 \\
HOMA- $\beta$ & $40.9 \pm 35.5$ & $131.5 \pm 90.7$ & 81.7 & 0.01 \\
\hline
\end{tabular}



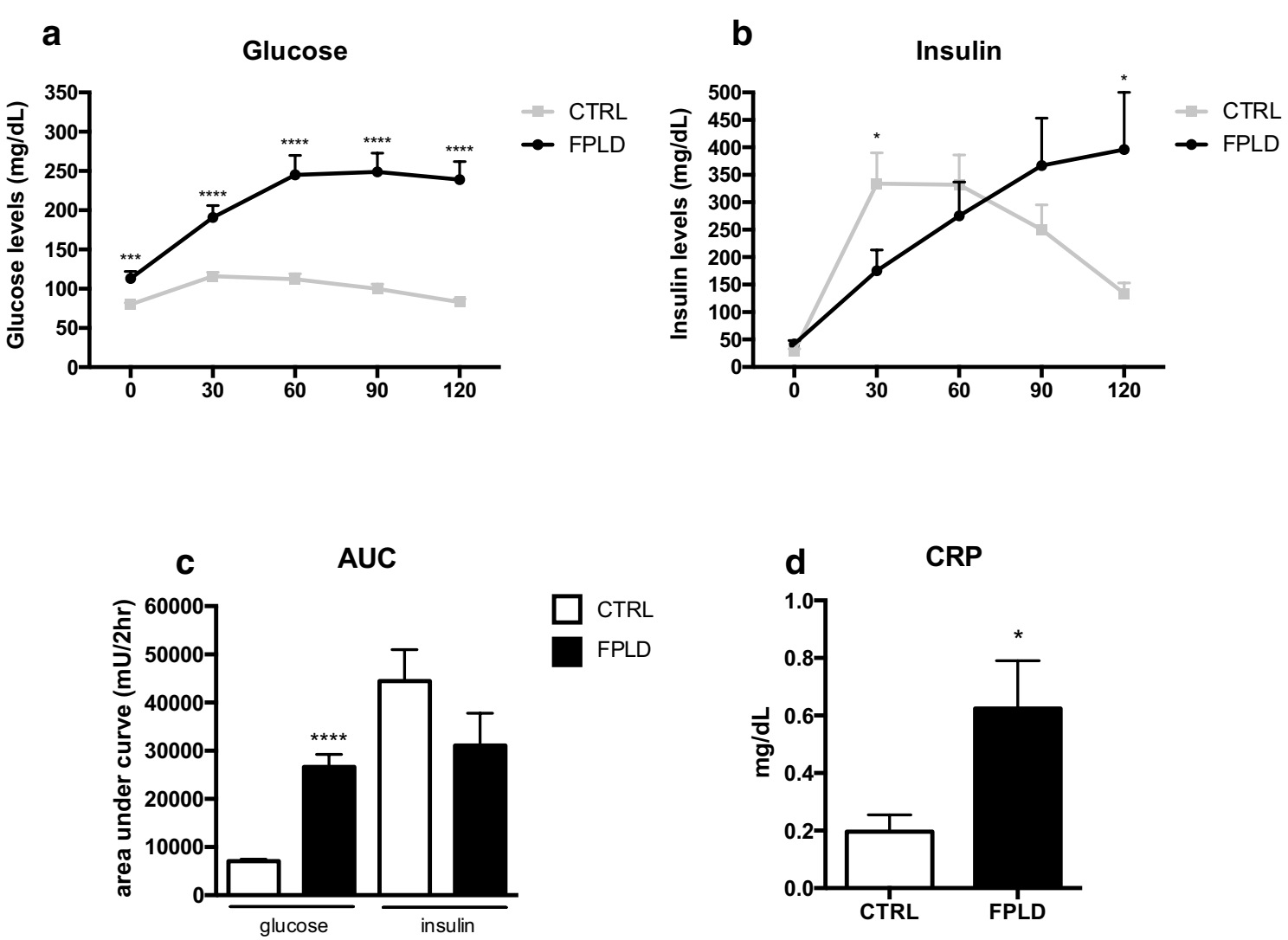

Fig. 2 Oral Glucose Tolerance Test demonstrates the uncontrolled glycemic regulation and reduced insulin secretion. a Graph showing glucose levels during OGTT. The individuals in the FPLD group presented increased glucose levels in compared to control group. ${ }^{* * *} p<0.005$, ${ }^{* * * *} p<0.001$ b Quantification of the insulin secretion during OTG. The FPLD group presented delayed insulin secretion compared to the control group * $<<0.05$. c Area under the curve of oGTT graphs (plasma glucose and insulin). The AUC of glucose graph showed that individuals of FPLD group have higher AUC in compared to control group, demonstrating the uncontrolled glycemic; however no observed differences in insulin AUC. Student's t test ${ }^{* * * *} p<0.001$. d Evaluation of serum C-Reactive Protein levels. Student's t test ${ }^{*} p<0.05$. Control group $(n=13)$ and FPLD group $(n=14)$
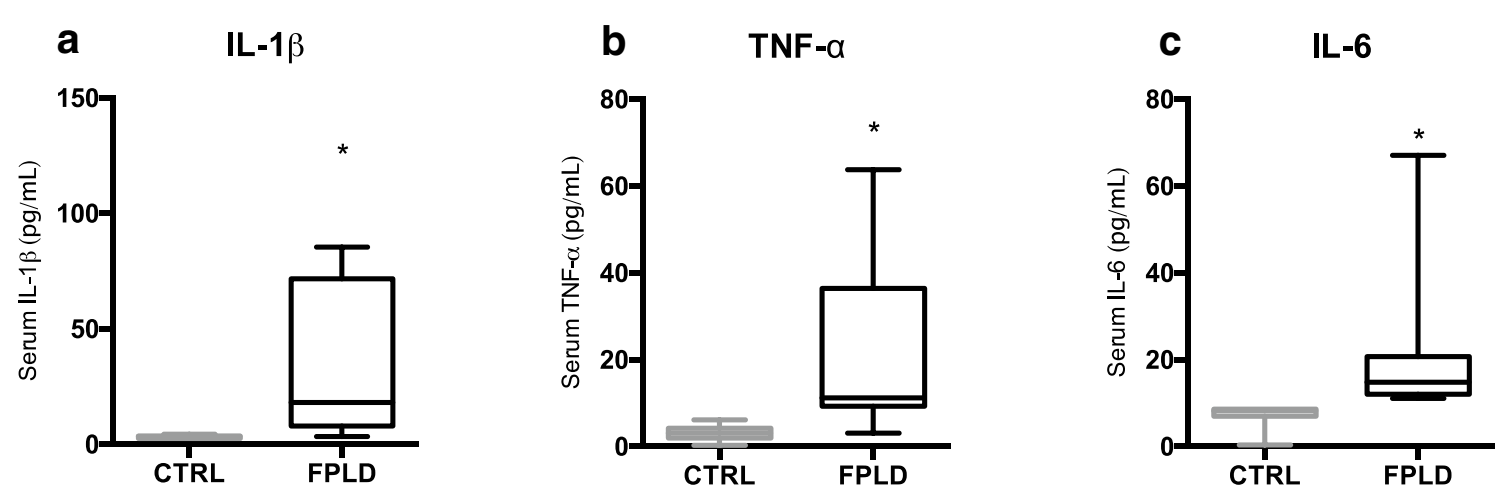

Fig. 3 Interleukins responsible for the inflammatory profile are increased in the serum of individuals with FPLD. The levels of the main interleukins inflammatory process are altered in the serum of patients with FPLD. a IL-1 $\beta$ quantification in the serum of individuals with FPLD and control subjects. Student's t test ${ }^{*} p<0.05$. b Serum TNF-a quantification of FPLD individuals and control. Student's t test ${ }^{*} p<0.05$. c Measurement of IL-6 concentration in the serum of FPLD individuals and control. Student's t test ${ }^{*} p<0.05$. Control group $(n=13)$ and FPLD group $(n=14)$

The scarcity of clinical and molecular studies involving individuals with FPLD can be justified by the fact that this is a pathological condition considered rare and therefore, there is great difficulty in finding people with FPLD and obtaining biological samples to be analyzed. Thus, in our study, the limited number of adipose tissue 


\section{Adipose tissue}

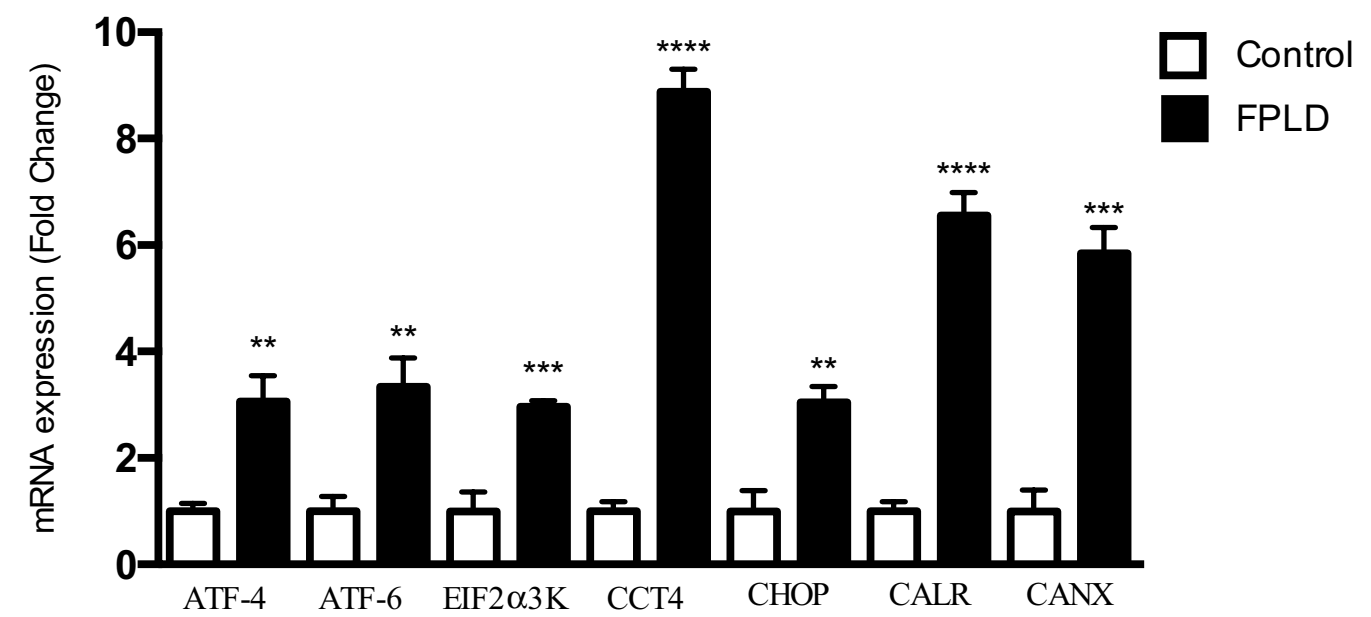

Fig. 4 Metabolic changes in adipose tissue in FPLD subjects. Gene expression in adipose tissue in response FPLD. Control group ( $n=4$ ) and FPLD group $(n=5)$, paired with the same age and BMI. Total RNA was analyzed by real-time qPCR; data represented mean \pm SEM. Student's t test **** $p<0.001$ versus control, ${ }^{* *} p<0.01,{ }^{* * *} p<0.005$

samples used for the analysis of gene expression was caused by the small number of patients who accepted to undergo the aesthetic procedure to obtain adipose tissue. However, due to the rare number of people with FPLD, it is believed that the analyzed samples may provide very important information for understanding FPLD and how it induces dyslipidemia and insulin resistance. Another limitation of our study is that molecular evaluation was performed on subcutaneous adipose tissue of the neck and, therefore, may not represent other sites of subcutaneous tissue, usually more involved with insulin resistance, inflammation and activation of ERS, such as the abdomen.

Insulin resistance (IR) is the common pathophysiological component in obesity, metabolic syndrome and particularly type 2 diabetes mellitus, leading to varying degrees of altered glucose tolerance and may be manifested clinically by hyperglycemia and/or hyperinsulinemia. Insulin with its metabolic effects or growth promoters is the main anabolic hormone in the human body. Its action, which depends on the correct binding of the hormone to its receptor on the plasma membrane of the cells, involves a complex response that affects the metabolism of lipids, protein and carbohydrate [19]. In the other hand, the reduction of body fat especially in cases of lipodystrophy, the presence of insulin resistance becomes a primary factor for metabolic manifestations of this disease. The FPLD is a heterogeneous group of genetic disorders characterized by marked loss of subcutaneous fat of the extremities [1]. Patients with lipodystrophy revealed phenotypes that can be linked to autosomal dominant or recessive mutations. Among the autosomal dominant mutations, three loci of candidate genes were identified: the gene encoding the nuclear lamin $\mathrm{A} / \mathrm{C}(L M N A)$, the gene of peroxisome proliferator activated receptor gamma (PPARG) and the gene encoding protein kinase $\mathrm{B} \mathrm{v}$-Akt-murine thymoma oncogene homolog 2 (AKT2) [20]. In this study, it was observed that individuals with FPLD had higher insulin resistance, as evidenced by HOMAIR calculation, as well as the calculation of $\mathrm{AUCg}$, obtained by oGTT. The estimate of IR and function of the pancreatic $\beta$ cells by the HOMA method is derived from a feedback model at baseline equilibrium condition [21]. The HOMA- $\beta$ and AUCi values, obtained by patients with lipodystrophy, prove inadequate insulin secretion capacity. The state of IR in normal situations determines the compensatory increase in insulin secretion by the pancreas, which can be observed in obesity, however, not always detects the development of glycemic alterations [22]. To evolve with abnormal glucose levels, as observed in diabetic patients and specifically in this case in individuals with FPLD, there must be a decrease in insulin secretion by the pancreas, resulting in a relative insulin deficiency, manifested by loss glucose tolerance $[19,23]$.

Due to the simplicity of calculating the HOMA index, this has been increasingly used in clinical practice for the assessment of RI, requiring the determination of the cut-off points of the population, for example, the cut-off point defined by the BRAMS study [24], which evaluated Brazilian individuals of São Paulo State, detecting 
that the cutoff point for this population is 2.7 for the diagnosis of IR. The HOMA-IR of patients with lipodystrophy showed values below the cutoff point suggested by the Brazilian study, and since there are no cuts of HOMA-IR and HOMA- $\beta$ for patients with this disease, we used the comparison with the control group, composed of individuals which showed no alterations in the distribution of body fat, observing that the group of patients with FPLD had higher IR and less secretion of capacity by pancreatic $\beta$ cells. Corroborating this data, individuals with FPLD have metabolic disorders that induce an increase in triglyceride levels, fasting glycemia and low levels of HDL, which are diagnostic criteria for the metabolic syndrome.

The IR states are accompanied by lipotoxicity and secretion of adipokines [25,26], which converge towards the development of metabolic inflammation [27]. The DM proinflammatory state is well known, being the adipose tissue adipokines potent inflammatory mediators [28] and sensitive to stimuli influenced by metabolic control [29-32]. In our results lipodystrophy patients did not show a systemic inflammatory state, when we evaluated the CRP levels. However, since we did not evaluate the ultrasensitive CRP, which is related to the development of cardiovascular diseases [33], we believe that the normal levels of CRP seen in patients with FPFD can be explained by the fact that we did not perform more sophisticated dosages which are usually used by other authors. Though, in order to deepen our studies on the inflammatory profile of lipodystrophy we analyzed the plasma levels of cytokines involved in the development of IR such as IL-1 $\beta$, IL-6 e TNF- $\alpha[28,34]$. Our data show that individuals with FPLD develop chronic and subclinical systemic inflammation due to elevations in IL-1 $\beta$, TNF- $\alpha$ and IL- 6 concentrations. Thus, our data suggest that the increased inflammatory process induced by these cytokines could contribute to the exacerbation of insulin resistance in individuals with FPLD.

Hyperglycemia and increase of inflammatory cytokines present in DM, can induce activation of the ERS in various cell types, including the pancreatic beta cells, triggering their apoptosis [35]. The ER has multiple cellular functions mainly folding, assembly and formation of disulfide bonds of proteins. The Unfolded Protein Response (UPR) is a mechanism that is activated by the high concentration of proteins in the ER resulting immature protein degradation, decreasing the translation rate, and the increase of chaperones. Our data on gene expression of key proteins related to ERS in adipose tissue of individuals with FPLD demonstrated the hyperactivation of the UPR. These data suggest that the existence of molecular and metabolic disorders in adipocytes of individuals with FPLD is associated with the modulation of ERS, which may explain the insulin resistance and hyperglycemia in these patients.

The major membrane proteins of the ER involved in induction of UPR such as eIF2a3K (RNA-activated protein Kinase-like ER Kinase) and ATF-6 (Activating Transcription Factor 6) [36], showed increased gene expression in adipocytes of patients with FPLD characterizing the state of ERS. PERK activation in the UPR results in inhibition of translation of mRNAs, but paradoxically some mRNAs encoding proteins of adaptation to stress become relevant such as ATF-4 (Activating Transcription Factor 4) [37]. Furthermore activation ATF-4 by PERK pathway leads to increased expression of $\mathrm{CHOP}$ that retains the cell cycle and induce apoptosis [38].

The increase in expression of calreticulin and calnexin can be explained by the imbalance caused by the differences in intracellular calcium concentration that affects the secretory machinery of the ER [12]. In the case of increased expression of CCT4 (Chaperonin Containing TCP1 subunit 4) in adipose tissue of patients with FPLD it can be characterized by the necessity of raising the recovery rate of malformed proteins due to ERS, because these chaperones have the function of assisting the correct folding of proteins by ATP hydrolysis [39, 40]. These chaperones are also necessary for the folding of tubulin and actin in cells [41] besides being connected directly to many newly synthesized polypeptides in mammalian cells [42].

Thus, the activation of ERS in adipose tissue of patients with FPLD could be responsible for the organic response to metabolic stress inducing a severe metabolic syndrome seen in these patients compared to healthy control subjects. To our knowledge, this is the first study evaluating the ERS activation in FPLD patients, but since we studied a small sample, besides FPLD is a rare clinical situation, our findings shall be confirmed with further studies.

\section{Conclusions}

In conclusion, this study demonstrated that Familial Partial Lipodystrophy of the Dunnigan type induces important metabolic disorders such as hyperglycemia, insulin resistance and hypertriglyceridemia. These damaging clinical environment are associated to increased systemic inflammatory profile and activation of the ERS in the subcutaneous adipose tissue, supporting the importance of fat deposits in the correct places of the human body. The discoveries of the findings may provide new routes to innovation of treatment providing a new perspective for the control of metabolic syndrome in this pathology. 


\section{Additional files}

Additional file 1: Table S1. Primers sequence.

Additional file 2: Figure S1. Human Unfolded Protein Response PCR-Array Layout in adipose tissue in individuals with FPLD. Regulation of genes expression in adipose tissue in response FPLD. (A) Heatmap representing the quantitation of 84 genes of ERS expressed in the subcutaneous adipose tissue of control group and FPLD group. (B) Quantitation of 84 genes (Fold-Change) in subcutaneous fat. ERS important genes are over-expressed in adipocytes of individuals with FPLD. Total RNA from adipose tissue were isolated from control group $(n=4)$ and FPLD group $(n=5)$ as described in the Experimental Procedures.

\section{Abbreviations}

FPLD: familial partial lipodystrophy of the Dunnigan type; HbA1c: glycated hemoglobin; LDL-C: lower-density lipoprotein cholesterol; HDL-C: high-density lipoprotein-cholesterol; TG: triglyceride; AUC: area under curve; OGTT: oral glucose tolerance test; ERS: endoplasmic reticulum stress; ATF-4: activating transcription factor 4; ATF-6: activating transcription factor 6; EIF2a3K: eukaryotic translation initiation factor 2-alpha kinase 3; CCT4: chaperonin containing TCP1 subunit 4; CHOP: C/EBP Homologous Protein; CALR: calreticulin; CANX: calnexin; IL-1 $\beta$ : interleukin 1 beta; TNF-a: tumor necrosis factor alpha; IL-6: interleucina 6; DM: diabetes mellitus; HOMA-IR: homeostasis model of assessment insulin resistance; HOMA- $\beta$ : homeostasis model of assessment $\beta$ cells function.

\section{Authors' contributions}

MCFF and MCF coordinated the study. MCFF and RCF planned the experiments, performed the study and drafted the manuscript. LZM and PMG performed the clinical evaluation. RI and LCCF performed the biopsies. All authors contributed to the interpretation of results. All authors read and approved the final manuscript.

\section{Author details}

${ }^{1}$ Department of Medicine, Division of Endocrinology and Metabolism, School of Medicine of Ribeirao Preto, University of Sao Paulo, Ribeirao Preto, São Paulo, Brazil. ${ }^{2}$ University of Brasilia-UNB, Federal District, Brasilia, Brazil. ${ }^{3}$ Department of Opthalmology, Otolaryngology, Head and Neck Surgery, School of Medicine of Ribeirao Preto, University of Sao Paulo, Ribeirao Preto, São Paulo, Brazil.

\section{Acknowledgements}

The authors are grateful to Maria Aparecida Nunes Ferreira and Sebastião L. Brandão Filho for technical assistance.

\section{Competing interests}

The authors declare that they have no competing interests.

\section{Availability of data and materials}

The authors will make full data available by request.

\section{Consent for publication}

Not applicable.

\section{Ethical approval and consent to participate}

The study was approved by the Research Ethics Committee of the University of São Paulo- Hospital of Medical School of Ribeirão Preto (HC-FMRP-USP)—Process HCRP no 6711/2012. All patients provided written consent to participate in the study.

\section{Funding source}

FAEPA.

\section{Publisher's Note}

Springer Nature remains neutral with regard to jurisdictional claims in published maps and institutional affiliations.
Received: 18 July 2017 Accepted: 16 December 2017

Published online: 09 February 2018

\section{References}

1. Garg A, Misra A. Lipodystrophies: rare disorders causing metabolic syndrome. Endocrinol Metab Clin North Am. 2004;33(2):305-31.

2. Garg A. Clinical review\#: lipodystrophies: genetic and acquired body fat disorders. J Clin Endocrinol Metab. 2011;96(11):3313-25.

3. Dinges $W L$, et al. Regional body fat distribution in HIV-infected patients with lipodystrophy. J Investig Med. 2005;53(1):15-25.

4. Beraldo RA, et al. Proposed ratios and cutoffs for the assessment of lipodystrophy in HIV-seropositive individuals. Eur J Clin Nutr. 2015;69(2):274-8.

5. Jacob KN, Garg A. Laminopathies: multisystem dystrophy syndromes. Mol Genet Metab. 2006;87(4):289-302.

6. Herbst KL, et al. Kobberling type of familial partial lipodystrophy: an underrecognized syndrome. Diabetes Care. 2003;26(6):1819-24

7. Donadille $\mathrm{B}$, et al. Etiological investigations in apparent type 2 diabetes: when to search for lamin A/C mutations? Diabetes Metab. 2005;31(6):527-32.

8. Ozcan U, et al. Endoplasmic reticulum stress links obesity, insulin action, and type 2 diabetes. Science. 2004;306(5695):457-61.

9. Ou HY, et al. Endoplasmic reticulum stress induces the expression of fetuin-A to develop insulin resistance. Endocrinology. 2012;153(7):2974-84

10. Kaufman RJ. Stress signaling from the lumen of the endoplasmic reticulum: coordination of gene transcriptional and translational controls. Genes Dev. 1999;13(10):1211-33.

11. Mori K. Tripartite management of unfolded proteins in the endoplasmic reticulum. Cell. 2000;101(5):451-4.

12. Coe H, Michalak M. Calcium binding chaperones of the endoplasmic reticulum. Gen Physiol Biophys. 2009;28(Spec No Focus):F96-103.

13. Harding HP, et al. Transcriptional and translational control in the Mammalian unfolded protein response. Annu Rev Cell Dev Biol. 2002;18:575-99.

14. Kaneto $H$, et al. Oxidative stress, ER stress, and the JNK pathway in type 2 diabetes. J Mol Med (Berl). 2005:83(6):429-39.

15. Sánchez $P$, et al. Age-related lipid metabolic signature in human LMNAlipodystrophic stem cell-derived adipocytes. J Clin Endocrinol Metab. 2015;100(7):E964-73

16. Zhang $Y$, et al. Echinacoside's nigrostriatal dopaminergic protection against 6-OHDA-Induced endoplasmic reticulum stress through reducing the accumulation of Seipin. J Cell Mol Med. 2017;21(12):3761-75.

17. Peraldi $P$, et al. Tumor necrosis factor (TNF)-alpha inhibits insulin signaling through stimulation of the p55 TNF receptor and activation of sphingomyelinase. J Biol Chem. 1996;271(22):13018-22.

18. Monteiro $L Z$, et al. Body fat distribution in women with familial partial lipodystrophy caused by mutation in the lamin A/C gene. Indian J Endocrinol Metab. 2012;16(1):136-8.

19. Haring $\mathrm{HU}$, et al. Interaction of the insulin receptor kinase with serine/ threonine kinases in vitro. J Cell Biochem. 1985;28(2):171-82.

20. Garg A, Agarwal AK. Lipodystrophies: disorders of adipose tissue biology. Biochim Biophys Acta. 2009;1791(6):507-13.

21. Matthews DR, et al. Homeostasis model assessment: insulin resistance and beta-cell function from fasting plasma glucose and insulin concentrations in man. Diabetologia. 1985;28(7):412-9.

22. Kahn CR. Knockout mice challenge our concepts of glucose homeostasis and the pathogenesis of diabetes. Exp Diabesity Res. 2003;4(3):169-82.

23. Benito M. Tissue-specificity of insulin action and resistance. Arch Physiol Biochem. 2011;117(3):96-104.

24. Geloneze B, et al. HOMA1-IR and HOMA2-IR indexes in identifying insulin resistance and metabolic syndrome: Brazilian Metabolic Syndrome Study (BRAMS). Arq Bras Endocrinol Metabol. 2009;53(2):281-7.

25. Sartipy P, Loskutoff DJ. Expression profiling identifies genes that continue to respond to insulin in adipocytes made insulin-resistant by treatment with tumor necrosis factor-alpha. J Biol Chem. 2003;278(52):52298-306.

26. Steppan CM, et al. The hormone resistin links obesity to diabetes. Nature. 2001:409(6818):307-12. 
27. McNelis JC, Olefsky JM. Macrophages, immunity, and metabolic disease. Immunity. 2014;41(1):36-48.

28. Hotamisligil GS, Shargill NS, Spiegelman BM. Adipose expression of tumor necrosis factor-alpha: direct role in obesity-linked insulin resistance. Science. 1993;259(5091):87-91.

29. Foss-Freitas MC, et al. Effect of metabolic control on the in vitro proliferation of peripheral blood mononuclear cells in type 1 and type 2 diabetic patients. Sao Paulo Med J. 2006;124(4):219-22.

30. Foss-Freitas MC, et al. In vitro TNF-alpha and IL-6 production by adherent peripheral blood mononuclear cells obtained from type 1 and type 2 diabetic patients evaluated according to the metabolic control. Ann NY Acad Sci. 2006;1079:177-80

31. Foss-Freitas MC, et al. Effect of metabolic control on interferon-gamma and interleukin-10 production by peripheral blood mononuclear cells from type 1 and type 2 diabetic patients. Braz J Med Biol Res 2007;40(5):671-7.

32. Foss-Freitas MC, et al. Effect of the glycemic control on intracellular cytokine production from peripheral blood mononuclear cells of type 1 and type 2 diabetic patients. Diabetes Res Clin Pract. 2008;82(3):329-34.

33. Lima LM, et al. High-sensitivity C-reactive protein in subjects with type 2 diabetes mellitus and/or high blood pressure. Arq Bras Endocrinol Metabol. 2007:51(6):956-60.

34. Shoelson SE, Lee J, Goldfine AB. Inflammation and insulin resistance. J Clin Invest. 2006;116(7):1793-801.
35. Scheuner D, Kaufman RJ. The unfolded protein response: a pathway that links insulin demand with beta-cell failure and diabetes. Endocr Rev. 2008;29(3):317-33.

36. Shen Y, et al. Essential role of STAT3 in postnatal survival and growth revealed by mice lacking STAT3 serine 727 phosphorylation. Mol Cell Biol. 2004;24(1):407-19.

37. Harding HP, et al. Diabetes mellitus and exocrine pancreatic dysfunction in perk-/- mice reveals a role for translational control in secretory cell survival. Mol Cell. 2001;7(6):1153-63.

38. Ron D. Translational control in the endoplasmic reticulum stress response J Clin Invest. 2002;110(10):1383-8.

39. Kubota H, Hynes G, Willison K. The chaperonin containing t-complex polypeptide 1 (TCP-1). Multisubunit machinery assisting in protein folding and assembly in the eukaryotic cytosol. Eur J Biochem. 1995;230(1):3-16.

40. Spiess C, et al. Mechanism of the eukaryotic chaperonin: protein folding in the chamber of secrets. Trends Cell Biol. 2004;14(11):598-604.

41. Sternlicht $\mathrm{H}$, et al. The t-complex polypeptide 1 complex is a chaperonin for tubulin and actin in vivo. Proc Natl Acad Sci U S A. 1993:90(20):9422-6.

42. Yam AY, et al. Defining the TRiC/CCT interactome links chaperonin function to stabilization of newly made proteins with complex topologies. Nat Struct Mol Biol. 2008;15(12):1255-62.

\section{Submit your next manuscript to BioMed Central and we will help you at every step:}

- We accept pre-submission inquiries

- Our selector tool helps you to find the most relevant journal

- We provide round the clock customer support

- Convenient online submission

- Thorough peer review

- Inclusion in PubMed and all major indexing services

- Maximum visibility for your research

Submit your manuscript at www.biomedcentral.com/submit 